\title{
Thresholds, switches and hysteresis in hydrology from the pedon to the catchment scale: a non-linear systems theory
}

\author{
J. Philip O'Kane and Denis Flynn \\ Boole Centre for Research in Informatics, Department of Civil and Environmental Engineering, UCC, Cork, Ireland \\ Email for corresponding author: p.okane@ucc.ie
}

\begin{abstract}
Hysteresis is a rate-independent non-linearity that is expressed through thresholds, switches, and branches. Exceedance of a threshold, or the occurrence of a turning point in the input, switches the output onto a particular output branch. Rate-independent branching on a very large set of switches with non-local memory is the central concept in the new definition of hysteresis. Hysteretic loops are a special case. A selfconsistent mathematical description of hydrological systems with hysteresis demands a new non-linear systems theory of adequate generality. The goal of this paper is to establish this and to show how this may be done. Two results are presented: a conceptual model for the hysteretic soil-moisture characteristic at the pedon scale and a hysteretic linear reservoir at the catchment scale. Both are based on the Preisach model. A result of particular significance is the demonstration that the independent domain model of the soil moisture characteristic due to Childs, Poulavassilis, Mualem and others, is equivalent to the Preisach hysteresis model of non-linear systems theory, a result reminiscent of the reduction of the theory of the unit hydrograph to linear systems theory in the 1950s. A significant reduction in the number of model parameters is also achieved. The new theory implies a change in modelling paradigm.
\end{abstract}

Keywords: hysteresis, multiphase flow, Preisach Model, rate-independence, switching, non-local memory, porous media

\section{Introduction}

\section{THRESHOLDS AND SWITCHES AT THE}

\section{CATCHMENT SCALE}

Thresholds and switches occur in many models of the hydrological cycle at the catchment scale. When a threshold value in a state variable is surpassed, a flow rate is switched on, and when the same state variable falls below that threshold, the flow is switched off. The mental picture of the model consists of bell-mouth inlets in water tanks, weirs and orifices with invert levels above the channel floor, and other similar devices of the hydraulic laboratory. In such cases the threshold values are usually the same for switching between 'on' and 'off', and the memory of such switches is local in time. In a system with local memory the past exerts its influence on the future through the current value of the output. More complex switches are possible.

\section{THRESHOLDS AND SWITCHES IN SOIL- ATMOSPHERE INTERACTION}

At the pedon scale of one metre, the hydrological interaction of soil moisture and the atmosphere is usually regarded as subject to control by two switches:

(1) an outer switch setting the direction of water flux, as evaporation, or evapo-transpiration, in the upward direction, or as precipitation in the downward direction, and

(2) an inner switch assigning the control of each flux to either the atmosphere or the soil, depending on the concentration of soil moisture in the upper layer.

In both cases the flux is initially under atmospheric control at the so-called potential or maximum rates set by the atmosphere.

The threshold for the outer switch is zero: at each point in time, either evaporation or precipitation occurs, but not both. In contrast, the threshold for the inner switch varies with the evolving dynamics of soil moisture. The moisture content in the top of the soil controls:

(1) the onset of ponding during precipitation, and 
(2) the onset of 'phase two' evaporation.

Ponding occurs when the soil reaches saturation at the surface and the saturated upper soil reduces the infiltration rate to a value less than the precipitation rate; in the case of 'phase two' evaporation, the flux drops below the potential rate because water starts to move in the vapour phase through a 'bottleneck' of variable thickness. This view is widely accepted for soil-atmosphere columns with a horizontal scale of one metre. At larger spatial scales a dynamic patchwork of switches in different states may occur (O'Kane, 1991). When a single-valued relationship is used to relate moisture content to water potential, these switched boundary conditions for the Philip-Richards equation describe a system with local memory where the past exerts its influence on the future through the current value of the output. However, the dynamics of soil moisture exhibits nonlocal memory. New models with non-local memory are required.

\section{THRESHOLDS AND SWITCHES IN SOIL MOISTURE}

The soil moisture characteristic for unsaturated soil relates water content to its potential. It is multi-valued. The thresholds for switching between the infinitely many branches of this characteristic — the so-called scanning curves of all orders - are reversals in the moisture content through local maxima or minima whenever they occur. Consequently, the current moisture content of a small sample of unsaturated soil may depend on many discrete turning points in its past. Future values depend not only on the current value of the output (potential) but also on past turning points in the input (water content) as well. A full turning point is a local maximum or minimum - contraflexure is excluded. In other words, the memory of the process is nonlocal. Smooth differential (and integro-differential) operators, such as the Philip-Richards equation, cannot model non-local memory.

\section{HYSTERESIS IN SOIL MOISTURE AT DIFFERENT SCALES}

The word hysteresis is of Greek origin and is derived from husteros meaning 'coming behind'. It was introduced into the scientific vocabulary about 120 years ago by the Scottish physicist Alfred Ewing as follows "when there are two quantities $M$ and $N$, such that cyclic variations of $N$ cause cyclic variation of $M$, then if the cyclic changes of $M$ lag behind those of $N$, we may say that there is hysteresis in the relation of $M$ and $N$ " (Ewing, 1895). Further historical details are presented in Cross and Allan (1988).

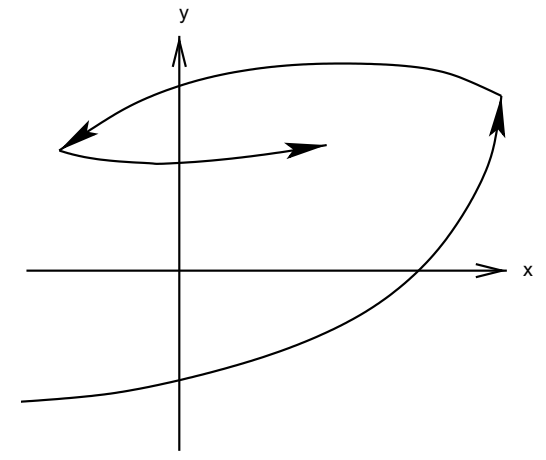

Fig. 1. Rate independent switching and branching.

Nowadays 'hysteresis' is a term in widespread use. For example, it is used in mechanics (plastic hysteresis; Krejci and Sprekels, 1999), physics (ferromagnetic hysteresis; Mayergoyz, 1991), chemistry (phase transitions; Brokate and Sprekels, 1996), hydrology and soil physics (soil moisture hysteresis; Parlange, 1980), and economics (shock analysis; Cross, 1995). In many scientific and general encyclopaedias the meaning of the word is illustrated with a picture such as Fig. 1 where $x$ is the input and $y$ the output.

Seventy-five years ago, Haines (1930) observed the phenomenon of hysteresis in unsaturated soils. It is believed, without adequate experimental verification, that (a) the hysteresis of soil-water is deterministic and rate-independent when considered on the time-scales of water flow, and (b) the fundamental mechanism is hysteresis in the contact angle of the air-water interface attached to the soil particles. As the flow of water changes direction the air-water-soil contact angle flips (de Gennes et al., 2003).

The observation that a catchment containing the same quantity of water behaves differently when charging or discharging water is sometimes regarded as evidence of hysteresis. Furthermore, the residual errors in hydrological predictions frequently show very high serial correlation, especially during periods of flow recession between storms. This suggests the presence of non-local memory.

The purpose of this paper is to provide a mathematically consistent non-linear theory of thresholds, switches and branches, collectively known as hysteresis, that may be applied to hydrological processes at different scales (O’Kane, 2004).

\section{Rate independent systems with switches and branches}

The concepts of rate-independent processes and systems with non-local memory were introduced, but not defined, in the previous section. These concepts are central to the discussion that follows. 


\section{SYSTEMS WITH LOCAL MEMORY}

The operation of a system $S$ is described symbolically by the relation $y(t)=T[x(t)]$ where $x(t)$ and $y(t)$ are input and output functions of time $t$ respectively. When the operation is linear and time-invariant the operation reduces to the convolution integral

$$
y(t)=\int_{-\infty}^{t} x(\tau) h(t-\tau) d \tau
$$

The function $h(t)$ is the impulse response of the system and may contain jumps and delta-functions. It includes as special cases, Green's functions that solve the initial value problems of linear ordinary and partial differential equations. All such systems have local memory. To prove this, split the integral at an arbitrary instant $t_{0}$, the present moment in time. The output at all future moments in time, $t>t_{0}$, is

$$
\begin{aligned}
y(t) & =\int_{-\infty}^{t_{0}} x(\tau) h\left(t_{0}-\tau\right) d \tau+\int_{t_{0}}^{t} x(\tau) h(t-\tau) d \tau \\
& =y\left(t_{0}\right)+\int_{t_{0}}^{t} x(\tau) h(t-\tau) d \tau, \quad t>t_{0}
\end{aligned}
$$

Clearly, future output $y\left(t>t_{0}\right)$ depends only on (a) the present output $y\left(t_{0}\right)$, and (b) future input $x\left(t>t_{0}\right)$. Past inputs $x(t<$ $t_{0}$ ), exert their influence on the future through the present output $y\left(t_{0}\right)$ and not in any other way. This is the definition of local memory in a system $S$.

More general systems may be described by non-linear convolution

$$
y(t)=\int_{-\infty}^{t} \int_{-\infty}^{t} \ldots \int_{-\infty}^{t} h\left(t-\tau_{1}, \ldots, t-\tau_{n}\right) \prod_{i=1}^{n} x\left(\tau_{i}\right) d \tau_{i}
$$

Splitting the multiple integral at $t_{0}<t$, shows that non-linear convolution and, by extension, an expansion in a Volterra series of convolution integrals also have local memory. Local memory is not the same as finite memory. If $h(t)=0$ for $t>$ $M, M$ is called the response time, or finite memory, of the impulse response.

Processes with after-effect, or hereditary processes, such as occur in the theory of plasticity, have non-local memory because past inputs continue to influence future outputs in ways other than through the present output.

The simplest examples of processes with non-local memory occur in rate independent systems, the subject matter of the next section. These are the building blocks of more complex hysteretic systems.

\section{RATE-INDEPENDENT SYSTEMS}

A system described by an operator

$$
T: y(t)=T[x(t)]
$$

is said to be rate-independent, if and only if, the same relationship holds when time is subjected to an affine transformation, i.e. when $t$ is replaced by $a t+b$ in both $x$ and $y$, the following test is an identity

$$
y(a t+b)=T[x(a t+b)]
$$

In other words, when the input is displaced and stretched in time, the output from a rate-independent system is displaced and stretched in time in exactly the same way.

Time $t^{\prime}$ on an affine clock corresponds to time $t$ on the original clock when $t^{\prime}=a t+b$. Affine time $t^{\prime}$ is slowed down or speeded up with respect to the original clock depending on whether $a<1$ or $a>1$ respectively. Conservation of the 'arrow of time' requires $a>0$. A restriction $b<0$ ensures the affine clock starts $\left(t^{\prime}=0\right)$ at a point in time $t=-b / a$ in the future.

The image under the rate-independent transformation $T$ of the input $x(a t+b)$ is the output $y(a t+b)$. Consequently, the parametric path $P$ in the $(x, y)$ plane is the same for all values of $a$ and $b$, including $a=1, b=0$, but the speed at which $P$ is traversed is different for each value of $a$.

Suppose the operation of the system is described by $T=c I$ where $I$ is the identity operator and $c$ is a constant i.e. $y(t)=c x(t)$. This system is rate independent because the image of the input $x(a t+b)$ under this $T$ is $c x(a t+b)$ which is identical to the output $y(t)$ after the affine transformation has been applied to its argument $t$. Time-invariant linear systems that are rate-independent reduce to a constant times the identity operator: $T=c I\left(\mathrm{O}^{\prime} \mathrm{Kane}, 2005\right)$. The only known exception is the Hilbert operator (Holland, 2005).

The path $P$ of a rate-independent linear system is a segment of a straight line in the $(x, y)$ plane of slope $c$ that extends through the origin. If sudden jumps in $c$ occur, for example, at full-turning points in $x(t)$, or at thresholds on the $x$ axis, the system exhibits rate-independent switching on a cone of line segments, or linear branches, in the $(x, y)$ plane. This switching and branching may have local or non-local memory.

More general rate-independent operators may be represented by a piece-wise smooth, multi-branch relationship between the input and output in the $(x, y)$ plane. This is illustrated in Fig. 1.

In Fig. 1 when an input $x(t)$ is increasing, the output $y(t)$ follows a certain path, but on changing the direction of the input, the output does not return on the same path. The input $x(t)$ first passes through a local turning point, a maximum in the $t$-domain. This is followed by a minimum in the $t$ domain. These turning points select different branches of the rate-independent $(x, y)$ relationship. Reversal of the branch is a consequence of the turning in time of the input 
function $x(t)$. Jumps in $y$ are allowed at the turning points - not shown here. Rate independence asserts that the projection of $x(t)$ and $y(t)$ into the $(x, y)$ plane is always the same for all strictly monotonic transformations of time, i.e. all possible speeding up and slowing down of the process.

\section{THE HYSTERON WITH LOCAL MEMORY}

The dynamics of switching and branching in the $(x, y)$ plane may be simple or complex. When there are one or two possible branches through every point in the allowable domain of the $(x, y)$ plane, the system exhibits local memory. The simplest possible rate-independent model that exhibits switching and branching with local memory is the hysteron, the 'atom of hysteresis', where two different thresholds on the input $x(t)$ switch the output $y(t)$ between two values, 0 or 1 (see Fig. 2). Each threshold triggers a switch in the output when the input passes through the threshold in the appropriate direction. Passing through the threshold in the reverse direction triggers no change in output. In other words, the output is two-valued between the thresholds and single-valued to each side. Consequently, there are four branches, each represented by a line segment in the $(x, y)$ plane. The two horizontal branches are half-lines through $y=0$ and $y=1$ respectively with overlapping ends. Each end is marked by the threshold on the $x$-axis where a jump occurs to the other branch. The vertical branches are line segments of unit length located at the thresholds on the $x$ axis and connect the horizontal branches to form a rectangle with two 'tails'. Reversals in the input $x(t)$ across both thresholds generates a loop in the $(x, y)$ plane. The loop may be clockwise or anticlockwise around the rectangle.

\section{SWITCHING AND BRANCHING WITH NON-LOCAL} MEMORY

When there is a large, or infinitely large, number of possible branches through every point in the allowable domain of the $(x, y)$ plane, the current value of the output and the future input is insufficient to select a unique future branch. This is the case of non-local memory. The distinction is analogous

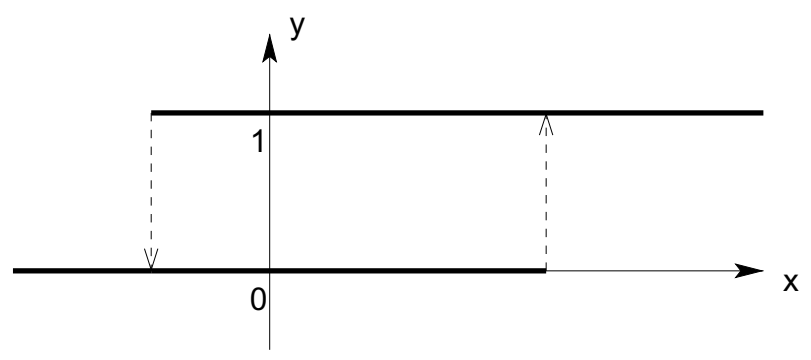

Fig. 2. The hysteron - the atom of hysteresis (anti-clockwise case). to that between Markovian and non-Markovian stochastic processes.

A rate-independent operator with switching and branching and non-local memory may model part of a hydrological system. Since it is part of a larger system, its input is not known in advance but is determined by its interaction with the rest of the hydrological system. Consequently, it is impossible to specify in advance the branch that will be followed in a particular realisation of the system dynamics.

\section{Hysteresis: theory and application}

\section{A NON-LINEAR SYSTEMS THEORY}

The collective name for these strongly non-linear phenomena is hysteresis. New mathematical concepts and analytical and numerical tools for treating rate-independent hysteresis have recently been developed. The modern mathematical concept of hysteresis was suggested by M. Krasnosel'skii and his co-workers (Krasnosel'skii and Pokrovskii, 1989: see also Visintin, 1994; Brokate and Sprekels, 1996; Krejci, 1996; Mayergoyz, 2003). New rate independent models have been developed that:

(1) detect and place in temporal order the full turning points of the input function, and

(2) use them to select the appropriate branches of the hysteresis non-linearity.

Without such models, a self-consistent mathematical description of hydrological systems with hysteresis is virtually impossible. The goal of this paper is to establish this and to show how this may be done. Two results are presented: a conceptual model for the hysteretic soilmoisture characteristic at the pedon scale and a hysteretic linear reservoir at the catchment scale. Both are based on the Preisach model. The Preisach model of hysteresis is built from an infinite number of hysterons connected in parallel. It is rate-independent and exhibits non-local memory.

A result of particular significance is the demonstration in this paper that the independent domain model of the soil moisture characteristic due to Childs, Poulavassilis, Mualem and others, is equivalent to the Preisach hysteresis model of non-linear systems theory, and is reminiscent of the reduction of the theory of the unit hydrograph to linear systems theory in the 1950s (Dooge and O'Kane, 2003). A significant reduction in the number of model parameters is also achieved. It also clarifies how hysteresis may be introduced into (1) conceptual hydrological models, and (2) the Philip-Richards model, with complete mathematical consistency. 


\section{HYSTERESIS IN HYDROLOGY}

Rate-dependent hysteresis has been discussed in hydrology for a long time, for example, in the looped-rating curve, and in similar plots of state and rate variables from systems of differential equations that model hydrological processes. These rate-dependent loops do not show affine similarity with respect to time, i.e. when the time argument of an input function is stretched, or speeded up, the corresponding output function is not stretched in the same way. For example, the attenuation of a sinusoidal input to a linear system depends on its frequency. Stretching the frequency changes the attenuation. Hence, any looped plot in the $(x, y)$ phase plane is rate dependent. In contrast to this, rateindependent hysteretic loops exhibit affine similarity with respect to time, i.e. when the time argument of an input function is stretched, the corresponding output function is stretched in the same way. No smooth differential or integrodifferential operator has this property. Neither can this kind of hysteresis be represented by a Volterra series of convolution integrals (O'Kane, 2005).

\section{HYSTERESIS IN SOIL PHYSICS}

In the literature on soil physics, the phenomenon of hysteresis is closely associated with the formation of loops. Hysteretic looping in the $(x, y)$ plane occurs when the input $x(t)$ returns to an extremum of the same kind, i.e. from local maximum to local maximum, or from local minimum to local minimum. Closed loops occur only under special circumstances. History-dependent branching on a very large set of switches is the central concept in the new definition of hysteresis, with looping taking its place as a special case.

\section{THE NON-LINEARITY OF HYSTERESIS}

Hysteresis is a non-linearity that is expressed through thresholds, switches and branches. Exceedance of a threshold switches the output onto a particular output branch. Fixed thresholds make hysteresis a non-linear process. In a linear system, doubling the input function doubles the output function. If the input to a hysteretic system is doubled, the output is not doubled, because the set of thresholds that are exceeded is different in each case. Consequently, the set of branches that is followed by the output is different in each case, and strict doubling of the output is impossible.

The test for linear superposition is also violated when the full turning points of the input function switch the output to particular branches. Consider two sinusoidal input functions of different frequencies and any linear combination of the two. All three input functions have different sets of full turning points. Consequently, the branches of the output function that are selected at full turning points will also be different in each case and the superposition test fails.

There is no contradiction between selection by threshold or full turning point. It will be seen subsequently in the Preisach model of hysteresis that it is the full turning points in the input function select sets of thresholds that are exceeded, rather than one individual threshold that is exceeded. The output from any set of branches must then be found by a weighted integration over the corresponding set of thresholds. This set has a non-local memory since it is defined by the turning points in the input function.

\section{The Preisach Model}

\section{THE HYSTERON, THE ATOM OF HYSTERESIS}

The simplest hysteretic system is the hysteron shown in Fig. 2 . Its operation is denoted by an operator $R_{\alpha, \beta}$. The input to $R, x(t)$, is compared to two threshold values $\alpha<\beta$. The output from $R, y(t)$, can take one of two values, 0 or 1 , depending on the history of $x(t)$ as it crosses the thresholds; at any moment in time the hysteron is either switched 'on' or 'off'. In Fig. 2, the bold lines represent the set of possible inputoutput pairs. They overlap in the interval $\alpha<x<\beta$. If the input is increasing and crosses the lower threshold, the output remains at 0 . If the input increases further and crosses the upper threshold, the output jumps from 0 to 1 . If the input then decreases, the jump back to 0 occurs when the input crosses the lower threshold, completing an anticlockwise hysteretic loop that is rate-independent.

The output $y(t)$

$$
y(t)=R_{\alpha, \beta}\left[t_{0}, \eta_{0}\right] x(t), \quad t \geq t_{0},
$$

depends on the input $x(t), t \geq t_{0}$, and on the initial state $\eta_{0}$. Here the input is an arbitrary continuous scalar function; $\eta_{0}$ is either 0 or 1 . The scalar function $y(t)$ has at most a finite number of jumps on any finite interval $t_{0} \leq \mathrm{t} \leq t_{1}$.

The output behaves rather 'lazily': it prefers to be unchanged, as long as the phase pair $x(t), y(t)$ belongs to the union of the bold lines in the Fig 2. The value of Eqn. (1) at the moment $t$ is defined by the following explicit formula:

$$
\begin{aligned}
& y(t)=R_{\alpha, \beta}\left[t_{0}, \eta_{0}\right] x(t)= \\
& = \begin{cases}\eta_{0}, & \text { if } \alpha<x(\tau)<\beta \text { for all } \tau \in\left[t_{0}, t\right] ; \\
1, & \text { if there exists } t_{1} \in\left[t_{0}, t\right] \text { such that } \\
& x\left(t_{1}\right) \geq \beta, x(\tau)>\alpha \text { for all } \tau \in\left[t_{1}, t\right] ; \\
0, & \text { if there exists } t_{1} \in\left[t_{0}, t\right] \text { such that } \\
& x\left(t_{1}\right) \leq \alpha, x(\tau)<\beta \text { for all } \tau \in\left[t_{1}, t\right] .\end{cases}
\end{aligned}
$$


Equation (2) can be read as follows:

(1) Condition 1 states that as long as $x(\tau)$ remains within the threshold values $\alpha$ and $\beta$, for all times $\tau$ from the present time $t_{0}$ to some future time $t$, then the output $y(t)$ of the hysteron will remain at its initial state $\eta_{0}$, which can be either 0 or 1 .

(2). Condition 2 states that if there is a time $t_{1}$ before a future time $t$, such that the input $x(t)$ moves to the right of the $\beta$ threshold in Fig.(2) and $x(t)$ does not cross $\alpha$ threshold after time $t_{1}$, then the output will become 1 at time $t_{1}$ and remain there.

(3) The third and final condition states that if there is a time $t_{1}$ before a future time $t$, such that the input $x(t)$ moves to the left of the $\alpha$ threshold in Fig.(2) and $x(t)$ does not cross the $\beta$ threshold after time $t_{1}$, then the output will be 0 and remain there.

Section 28 (pages 262-273) of Krasnosel'skii and Pokrovskii (1989) presents the mathematical properties of this hysteron under the headings of 'ideal and non-ideal relays', terms in use in electrical engineering. Two of these properties are:

\section{- $\quad$ Property 1: Exclusion of superfluous switching}

The values of the output $y(t)$, corresponding to the continuous input $\left(t \geq t_{0}\right)$ to an anticlockwise hysteron $(\alpha<\beta)$ can be completely characterized on a closed interval of time $\left[\mathrm{t}_{1}, \mathrm{t}_{2}\right]$ by the following rule:

if either $y\left(t_{1}\right)=0$ and $x(t)<\beta$ for $t$ in that interval, or $y\left(t_{1}\right)=1$ and $x(t)>\alpha$ for $t$ in that interval, then $y(t)$ is constant in that interval $\left[\mathrm{t}_{1}, \mathrm{t}_{2}\right]$ and is equal to $y\left(t_{1}\right)$. Consequently, the output is piece-wise constant for $(t \geq$ $\left.t_{0}\right)$.

- Property 2: Monotonicity with respect to inputs Let $\left\{x^{\prime}(t), \eta_{0}^{\prime}\right\}$ and $\left\{x^{\prime \prime}(t), \eta^{\prime \prime}{ }_{0}\right\}$ be two different inputs and initial states of the hysteron. If they are monotonic with respect to each other:

$$
\eta^{\prime}{ }_{0} \leq \eta^{\prime \prime}{ }_{0} \text { and } x^{\prime}(t) \leq x^{\prime \prime}(t),\left(t \geq t_{0}\right)
$$

then the corresponding outputs are also monotonic with respect to each other:

$$
R_{\alpha, \beta}\left[t_{0}, \eta_{0}\right] x^{\prime}(t) \leq R_{\alpha, \beta}\left[t_{0}, \eta^{\prime \prime}{ }_{0}\right] x^{\prime \prime}(t),\left(t \geq t_{0}\right)
$$

The full turning points in the continuous input function $x(t)$ i.e. local maxima and minima with respect to time, divide $x(t)$ into monotonic pieces of two and only two types: increasing or decreasing with respect to time. Both types may contain points of contraflexure. This distinction simplifies the definition of the hysteron by separating it into two cases. These cases are used in the Preisach model below. The class of admissible input functions to the hysteron may be extended beyond piece-wise monotonic functions to include inputs with jumps at a finite number of discrete points in time. This case is not treated in this paper.

\section{THE PREISACH MODEL}

The Preisach Model was originally conceived to describe ferromagnetism (Preisach, 1935). It was subsequently found to be an excellent model for other phenomena (often due to the fundamental Mayergoyz Identification Theorem (Mayergoyz, 1991). The basis of the model is the following: Suppose there is a finite set of hysterons $R^{j}=R_{\alpha_{j}, \beta_{j}}, j=1, \ldots, N$. Now consider a parallel connection of these hysterons $R^{j}$ with the weights $\mu_{j}=\mu(j)$ $>0$. The output is then

$$
\begin{aligned}
& y(t)=y\left[t_{0}, \eta_{0}\right] \\
= & \sum_{j=1}^{N} \mu_{j} R^{j}\left[t_{0}, \eta_{0}(j)\right] x(t), t>t_{0} .
\end{aligned}
$$

This idea can be represented graphically as shown in Fig. 3 .

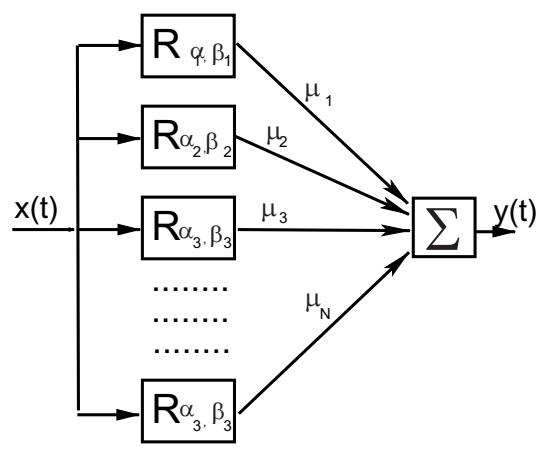

Fig. 3. Weighted parallel connection of a finite number of hysterons. The output $y(t)$ is determined by the weighted sum of all the hysterons and the input $x(t)$. Each hysteron is switched to either 0 or $I$ depending on the history of the system.

Hysterons can also be connected in series. The final outputs from $n$ hysterons in series or in parallel are fundamentally different, unlike linear sub-systems. Given $n$ linear sub-systems in series (or in parallel), the Equivalence Theorem (Dooge and O'Kane, 2003; pages 11-13), shows how to find the equivalent sub-systems in parallel (or in series).

The standard way to represent the Preisach model is with the Preisach $(\alpha, \beta)$ half-plane, where $\beta \geq \alpha$. Each point on the plane represents a hysteron. The coloured regions in Figs. 4 and 5 represent hysterons that are 'switched on'. In 


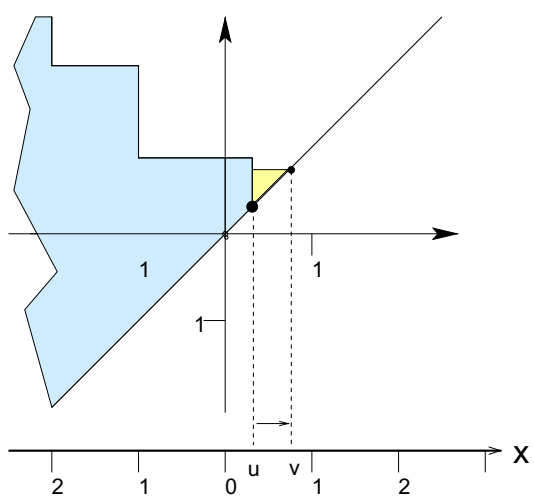

Fig. 4.

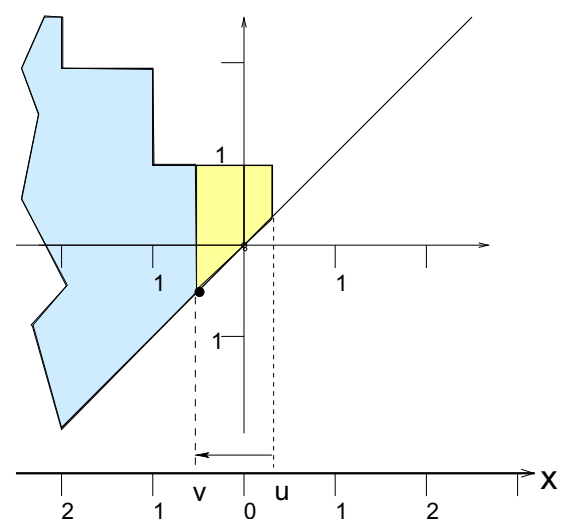

Fig. 5. The evolution of states on the Preisach plane.

the continuous case the parallel summation of the hysterons can be taken as the integral over the region where the hysterons are 'switched on' with some probability density. These figures illustrate the Preisach Model. Here the input $x(t)$ moves along the $\mathrm{x}$-axis, and controls the point on the diagonal above itself. The output $y(t)$ is the 'area' of the coloured domain with respect to some density.

The region that is integrated depends on the past history of the system. This history is recorded by a series of horizontal and vertical lines forming a staircase shape $L$ that can be seen in Figs. 4 and 5. The evolution of the 'staircase' $L$ is as follows: When an input $x(t)$ moves along the horizontal axis, it controls a point on the diagonal $\beta=\alpha$ above itself. When moving the point towards the upper right corner in Fig. 4, this point on the diagonal drags the horizontal line upwards, and colours in the domain between this horizontal line and the diagonal (this yellow triangle will be added to the region that is to be integrated). When moving the point towards the bottom left corner in Fig. 5, the diagonal point drags the vertical line to the left and decolours everything to the right of this line and above the diagonal (in moving from $\mathrm{u}$ to $\mathrm{v}$ the yellow region will be cleared and hence removed from the region that is to be integrated). The output $y(t)$ is the integral of the region defined by the blue domain with respect to some density. In other words, the staircase is defined by the full turning points in the input $x(t)$ : vertical risers are inserted in $L$ at local minima and horizontal treads at local maxima. The staircase $L(t)$ is the device, or mathematical object, for 'remembering' which hysterons are 'on' and which are 'off'. It has nonlocal memory. The reader is recommended to explore these ideas using the following java applets (Rasskazov, 2002) and (Flynn, 2003).

\section{PROPERTIES OF THE PREISACH MODEL}

Mayergoyz (2003) states the following properties of the nonlocal memory in the Preisach model (anticyclonic case) Wiping-out properties in $L(t)$ (also known as the return point memory)

- Each local maximum in the input $x(t)$ wipes out the vertices of $L(t)$ whose $\alpha$-coordinates are below this maximum, and each local minimum in $x(t)$ wipes out the vertices of $L(t)$ whose $\beta$-coordinates are above this minimum.

- Only the alternating series of dominant input extrema are stored by the Preisach model. All other input extrema are wiped out.

Congruency property of hysteretic loops

- All minor hysteresis loops in the $(x, y)$ plane corresponding to back-and-forth variations of inputs between the same two consecutive extremum values in $x(t)$ are congruent.

\section{Representation Theorem}

- The wiping-out property and the congruency property constitute the necessary and sufficient conditions for a hysteresis non-linearity to be represented by the Preisach model on the set of piece-wise monotonic inputs $x(t)$.

\section{REDUCTION OF THE INDEPENDENT DOMAIN} MODEL TO THE PREISACH MODEL

A half-plane, similar to the Preisach half-plane, can be found in the papers by Poulovassilis (1969, 1970a,b), Poulovassilis and Childs (1971), Poulovassilis and Tzimas (1974), Mualem (1974b, 1984), Mualem and Dagan (1975), Mualem 


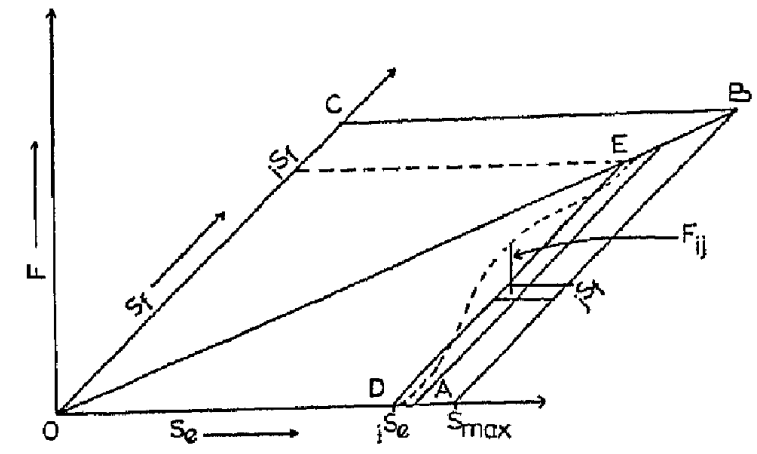

Fig. 6. A volume element above the area $\Delta s_{e} \times \Delta s_{f}$ on the half plane. Taken from (Poulovassilis, 1962).

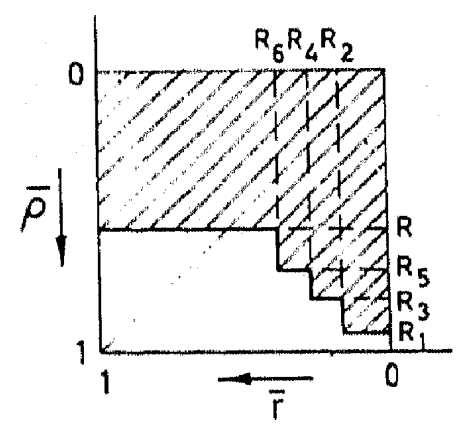

Fig. 7. Neel type diagram taken from (Mualem, 1974a). The notation $\bar{\rho}$ and $\bar{r}$ are the normalised radii of the pores and openings of the pores in the soil. This notation is equivalent to the alpha and $\beta$ notation in the Preisach Model. The notation to record the history of the turning points are $R_{1}-R_{6}$, where subscripts of odd values record the treads and the even subscripts record the risers of the staircase.

and Morelseytoux (1978), Mualem and Miller (1979) and others on the independent domain model. See for example Fig. 6 taken from Poulovassilis (1962).

However the absence in these papers of a figure similar to Fig. 5 shows that the concept of the output as a linear combination of a large number of hysterons, each with local memory acting in parallel, is not recognised explicitly.

A staircase similar to that of Fig. 5 is partly present as the necessary device, or mathematical object, for remembering the turning points in the input function as they switch sets of hysterons on and off; see, for example, Fig. 7 (Mualem's stairs attributed to Neel). The data structure required to represent the stairs in a digital machine is not present. Enderby's math object (see Eqn. 4) as used by Mualem, (1974a) is an attempt to define the required data structure.

$$
\theta\{\psi\}=\theta\left\{\begin{array}{llll} 
& \psi_{1} & & \psi \\
\psi_{0} & & \psi_{2} &
\end{array}\right\}
$$

These versions of the independent domain model are incomplete because they lack an explicit algorithm and data

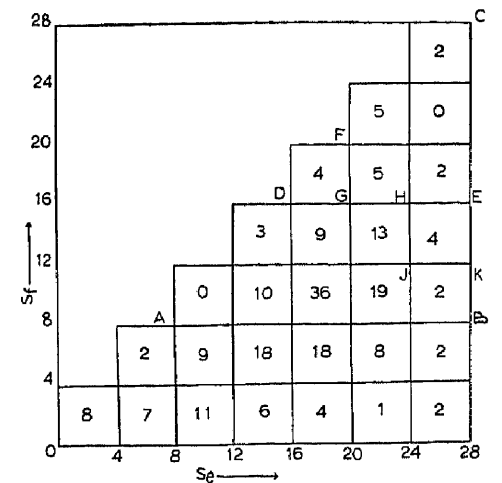

Fig. 8. Poulovassilis' discrete density diagram (Poulovassilis, 1962)

structures for generating the scanning curves of all orders. The central assumptions of rate-independence and non-local memory are overlooked, not just in these papers, but throughout the soil physics literature (Flynn and O'Kane, 2004,; Flynn, 2004; Flynn et al., 2005).

Figure 8 also shows a discrete two-dimensional representation of what is called in this paper, the Preisach density. Of necessity, it has a large number of parameters. A well chosen continuous density facilitates a reduction to none, one or two parameters, which is greatly to be desired, when attempting to solve the identification problem.

\section{APPLICATION OF THE PREISACH MODEL TO THE SOIL-MOISTURE CHARACTERISTIC}

All of the models mentioned above required many parameters to function: in fact, some of the models would require an infinite number of parameters. To achieve a reduction in the number of parameters it is necessary to use the Preisach model (Preisach, 1935; O'Kane et al., 2003a,b). Using this model as a basis, the fitting of experimental data was performed in Flynn (2004) and Flynn et al. (2005). In that paper, three models were defined, called the Zero, Beak and Wedge Models, which can be seen in Figs. 9, 11 and 13 respectively. Each of the models had a unique one-parameter, non-uniform density. The densities had the given form to match the van Genuchten equation and were defined by restricting the Preisach half-plane within different regions as shown in Figs. 9, 11 and 13. Because there was at most one parameter, a least-squares fitting could be used. The results from the fittings were much improved compared to Haverkamp et al. (2002). In fact, the Wedge model resulted in the best overall fits. The difference in the quality of the fits can be seen by comparing the fitting results for a given soil (soil no.23 from the GRIZZLY database (Haverkamp et al., 2002) in Figs. 10,12, and 14. For further details of the results of using the Preisach model to fit a series of soils see Flynn et al. (2005). 
(a)

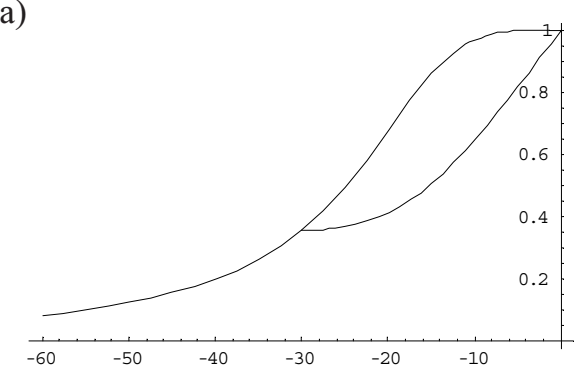

(c)

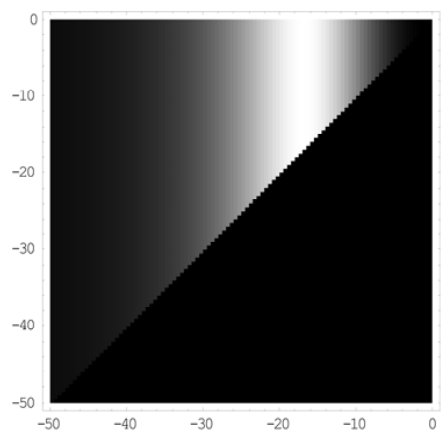

(b)

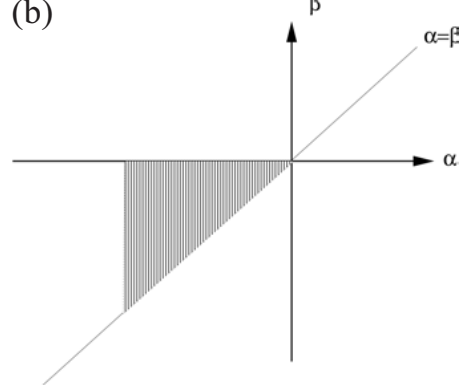

(d)

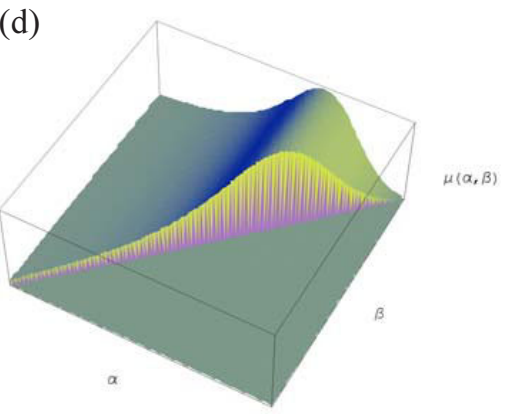

Fig. 9. Zero-parameter Model: (a) a sample hysteresis loop; (b) the measure support; (c) a density plot where the lighter shades correspond to a higher density; (d) a three-dimensional view where the height is proportional to the density.

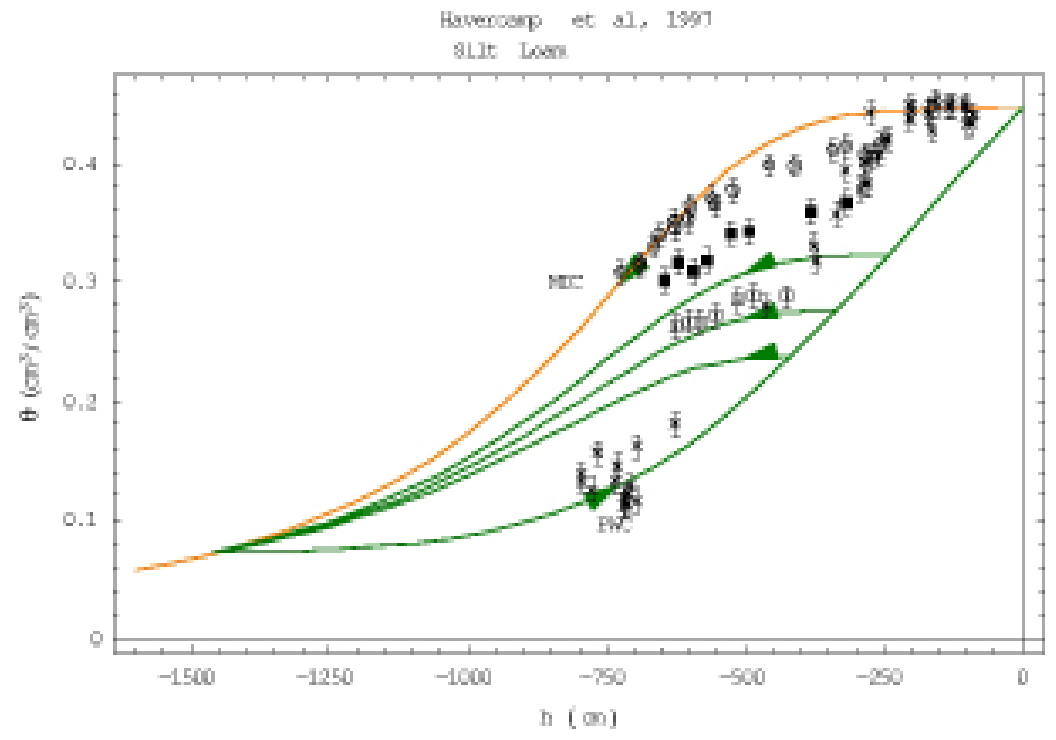

Fig. 10. Data sets for soil no. 23. Fitted using the zero-parameter model. Horizontal and vertical error bars correspond to the measurement errors in the soil pressure head and water content (i.e. $\pm 5 \mathrm{~cm}$ and $\pm 0.01 \mathrm{~cm}^{3} / \mathrm{cm}^{3}$ ).

\section{The modelling paradigm for hysteresis}

The linear reservoir is one of the simplest conceptual models at the catchment scale (O'Kane, 2005, 2006). It will serve to illustrate how hysteresis may be introduced into a much wider set of hydrological models. The modelling paradigm for hysteresis begins by separating the conservation laws from the rate-independent relationships that provide closure. The conservation laws have local memory but are rate dependent. Consequently, hysteresis cannot be introduced into them directly. The rate-independent relationships are 
(a)

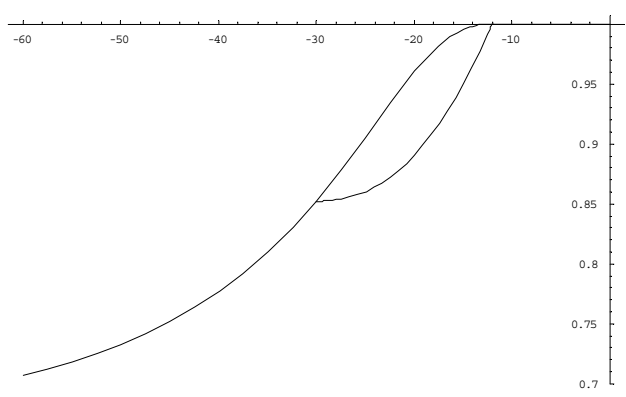

(c)

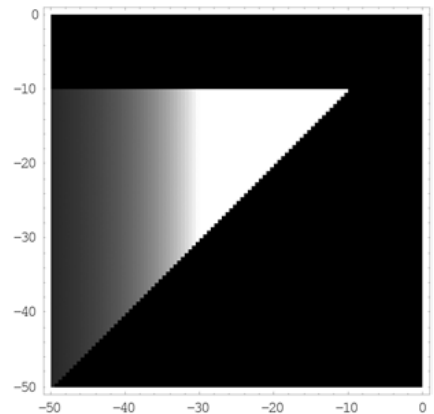

(b)

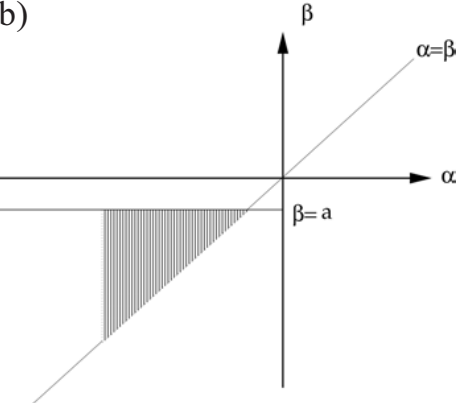

(d)

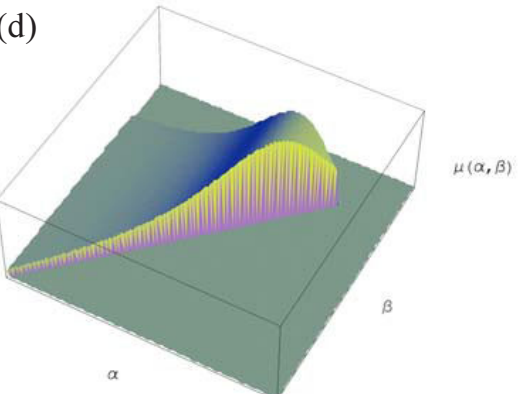

Fig. 11. The beak-model: (a) a sample hysteresis loop; (b) the measure support; (c) a density plot where the lighter shades correspond to a higher density; (d) a three-dimensional view where the height is proportional to the density.

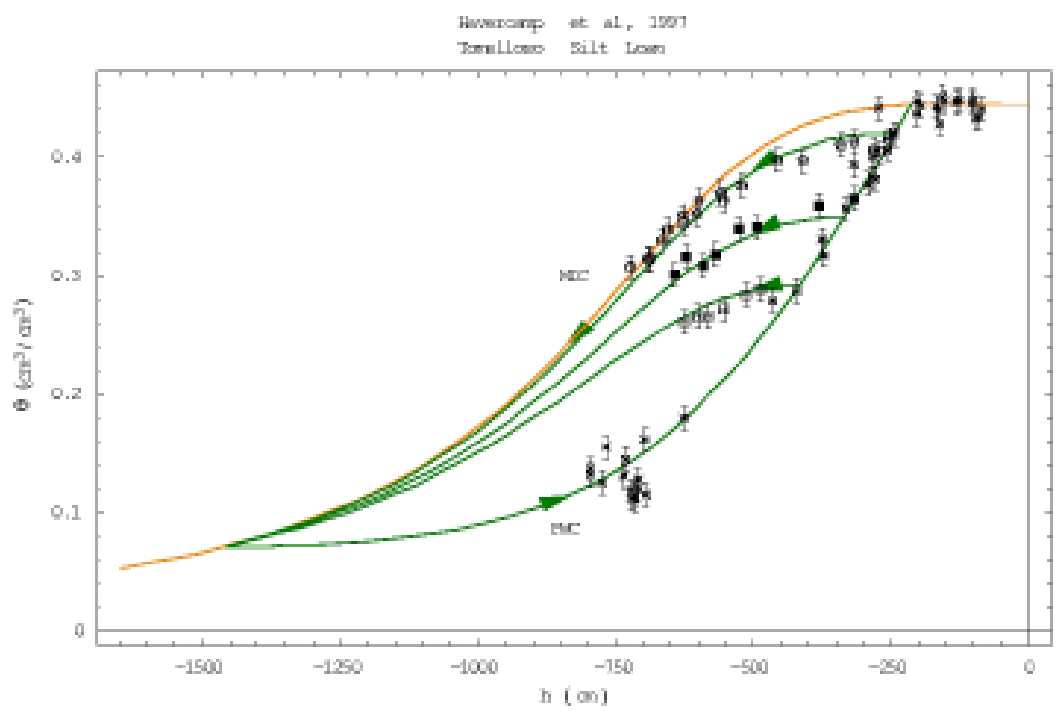

Fig. 12. Data set for soil no. 23 fitted using the Beak-model with fitted Parameter $a=-212.991$. Horizontal and vertical error bars correspond to the measurement errors in the soil pressure head and water content (i.e., $\pm 2 \mathrm{~cm}$ and $\pm 0.01 \mathrm{~cm}^{3} / \mathrm{cm}^{3}$

the locations where rate-independent hysteresis may be introduced with complete mathematical consistency. This hysteresis can have local or non-local memory. In the example to follow, a rate-independent Preisach operator with non-local memory is introduced into a linear reservoir in the only possible place in the model that ensures consistency.

\section{CONSERVATION OF WATER MASS - LOCAL}

\section{MEMORY}

The hydrological equation for a reservoir at any scale, and therefore at the catchment scale, is a conservation law

$$
\frac{d s}{d t}=x(t)-y(t), \quad s\left(t_{0}\right)=s_{0}
$$


(a)

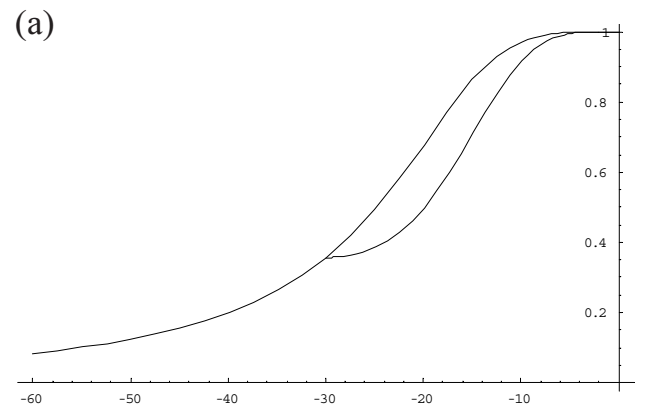

(c)

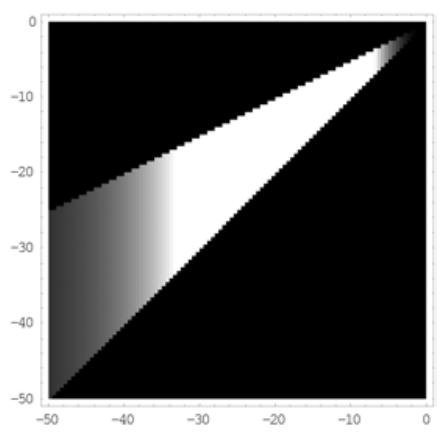

(b)

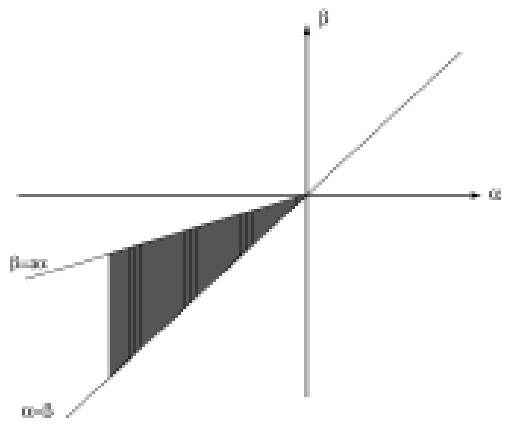

(d)

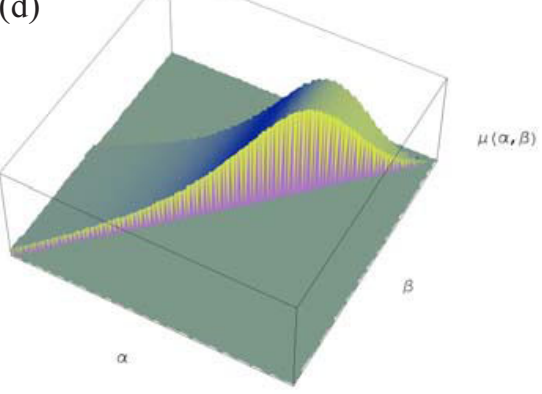

Fig. 13. The wedge-model with one parameter (a) a sample hysteresis loop; (b) the measure support; (c) a density plot where the lighter shades correspond to a higher density; (d) a three-dimensional view where the height is proportional to the density.

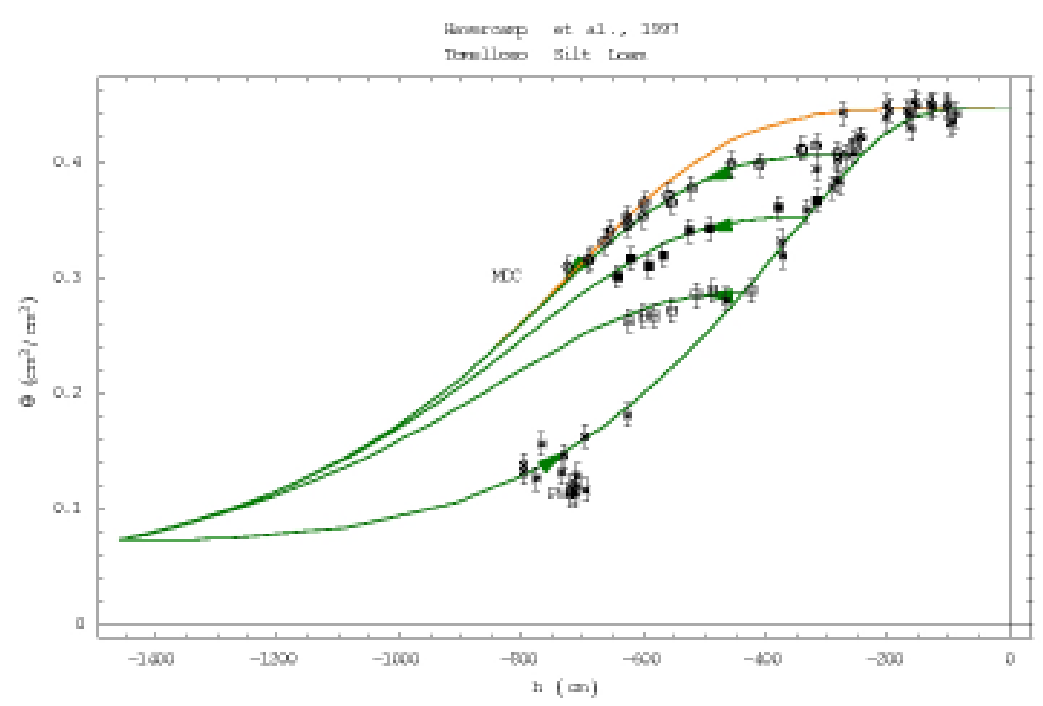

Fig. 14. Data sets for soil 23 fitted using the Wedge-model with fitted parameter ' $a$ ' $=0.428625$. Horizontal and vertical error bars correspond to the measurement errors in the soil pressure head and water content (i.e. $\pm 0.01 \mathrm{~cm}^{3} / \mathrm{cm}^{3}$.

where $s(t)$ is the instantaneous mass in storage at time $t$. Starting from a given initial condition $s_{0}$, the storage responds to the difference in the rates of inflow $x(t)$ and outflow $y(t)$. Whenever $x(t)=y(t)$, the storage $s(t)$ has a turning point. Full turning points are points where $s(t)$ is a local maximum $x=y, d x / d \mathrm{t}<d y / d t$, or minimum $x=y, d x / d \mathrm{t}$ $>d y / d t$, but is not a point of contraflexure $(x=y, d x / d \mathrm{t}=d y /$ $d t)$.
The integral form of this equation makes explicit the local memory of conservation

$$
s(t)=s\left(t_{0}\right)+\int_{t_{0}}^{t}(x(\tau)-y(\tau)) d \tau, t>t_{0}
$$

Past inputs $x\left(t<t_{0}\right)$, exert their influence on future storage $x\left(t>t_{0}\right)$ through the present storage $s\left(t_{0}\right)$ and not in any other way. 


\section{RATE INDEPENDENT CLOSURE - THE LINEAR RESERVOIR}

A second principle is required to close the dynamics of water storage. Functions $y=y(\mathrm{~s})$ provide closures that are rateindependent in the $(y, s)$ plane. The simplest case is a linear function

$$
y(t)=\frac{s(t)}{k}, \quad k>0 .
$$

The dynamics of this linear reservoir can be read as a single negative feedback-loop where $s(t)$, and consequently $y(t)$, are continuously adjusted to bring the output closer to the input. The speed and completeness of the adjustment is controlled by $k$. A feedback diagram in the graphical language of Forrester (1968) highlights the place where hysteresis may be included in the system dynamics (see Fig. 15).

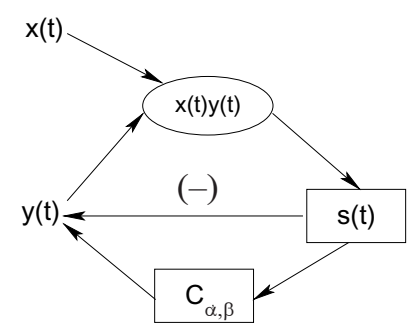

Fig. 15. Negative feedback loops in the hysteretic linear reservoir

\section{THE LINEAR RESERVOIR WITH ONE HYSTERON}

Hysteresis with local memory can be introduced by making $k$ a hysteron (O'Kane, 2005).

This can be represented in a feedback diagram, see Fig. 15.

In the interval $(\alpha, \beta)$ on the $s$ axis (Fig. 16), the output from the hysteron is either of two possible values of $k$

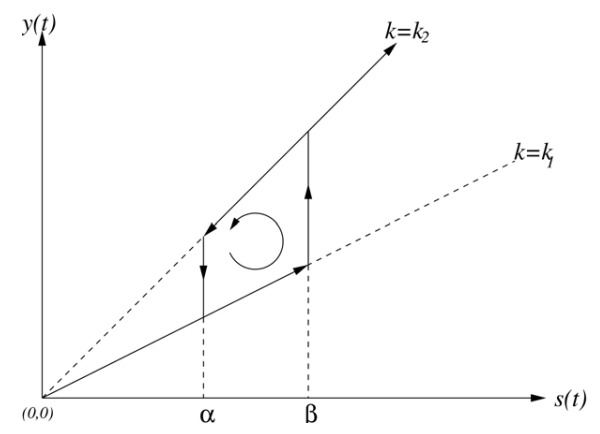

Fig. 16. A single hysteron on a cone. The cone is bounded by the lines $y(t)=\frac{s(t)}{k_{1}}$ and $y(t)=\frac{s(t)}{k_{2}}$. The thresholds $\alpha$ and $\beta$ are the thresholds of the cone. When $s(t)>\beta$ the output changes to $y(t)=\frac{s(t)}{k_{2}}$ and when $s(t)<\alpha$ the output becomes $y(t)=\frac{s(t)}{k_{1}}$. The direction of the loop is determined by the values of $k$ with respect to each other. In this case the loop is anticlockwise as $\frac{1}{k_{2}}>\frac{1}{k_{1}}$. depending on the history of $s(t)$. As $s(t)$ crosses the $\alpha$ or $\beta$ thresholds, $k$ may jump from one value to the other, generating a hysteretic loop on a cone through the origin in the $(y, s)$ plane. The slopes of the upper and lower lines of the cone are equal to the reciprocal values of the $k s$.

In the discrete case the output from a single cone-hysteron (Fig. 16) is the following

$$
y(t)=\left(C_{\alpha, \beta}\left[t_{0}, \eta_{0}\right] s(t)\right) s(t)
$$

where s(t) is the input to the system, and $C_{\alpha, \beta}$ is an operator which acts on $s(t)$ and is defined as

$$
\begin{aligned}
& C_{\alpha, \beta}\left[t_{0}, \eta_{0}\right] s(t)= \\
& = \begin{cases}\eta_{0}, & \text { if } \alpha<s(\tau)<\beta \text { for all } \tau \in\left[t_{0}, t\right] ; \\
\frac{1}{k_{2}}, & \text { if there exists } t_{1} \in\left[t_{0}, t\right] \text { such that } \\
\frac{1}{k_{1}}, & \text { if there exists } t_{1} \in\left[t_{0}, t\right] \text { such that } \\
& s\left(t_{1}\right) \leq \alpha, s(\tau)>\alpha \text { for all } \tau \in\left[t_{1}, t\right] ;\end{cases}
\end{aligned}
$$

where the constants $k_{1}$ and $k_{2}$ correspond to different lines of slope $\frac{1}{k_{1}}$ and $\frac{1}{k_{2}}$ respectively as shown in Fig. 16 and $\eta_{0}$ is the initial state, which can be either $\frac{1}{k_{1}}$ or $\frac{1}{k_{2}}$. Note that this definition is for a clockwise cone-hysteron when $\frac{1}{k_{1}}>\frac{1}{k_{2}}$ and an anti-clockwise cone-hysteron if $\frac{1}{k_{2}}>\frac{1}{k_{1}}$.

When integrating this operator-differential equation using an Euler scheme, this definition may be approximated as follows

$$
y_{\text {new }}=\frac{s(t)}{k_{\text {new }}}
$$

where $k_{\text {new }}$ is defined as

$$
k_{\text {new }}=\left\{\begin{array}{cc}
k_{2}, & s(t)>\beta \\
k_{1}, & s(t)<\alpha \\
k_{\text {old }}, & \alpha \leq s(t) \leq \beta
\end{array},\right.
$$

where the subscripts new and old refer to successive points in time in the numerical scheme; see also Krejci and Pokrovskii (2004)

\section{PARALLEL CONNECTION OF HYSTERONS ON A CONE.}

In the classical Preisach model a parallel connection of 


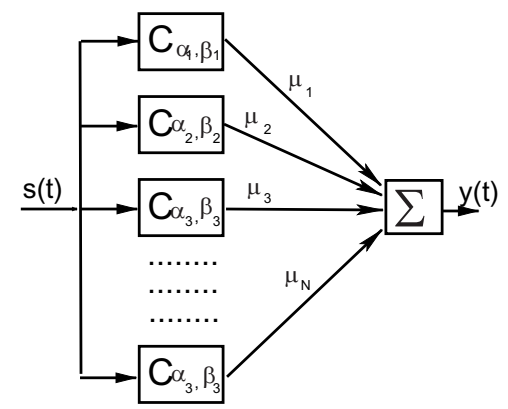

Fig. 17. A parallel connection of cone-hysterons.

weighted hysterons were summed to produce an output. Likewise a parallel connection of these hysterons can be taken as shown in Fig. 17, to produce the following equation

$$
\begin{aligned}
& y(t)=y\left[t_{0}, \eta_{0}\right](t) \\
& =\sum_{j=1}^{n} \mu_{j} C_{\alpha_{j}, \beta_{j}}\left[t_{0}, \eta_{0}\right] s(t), \quad t \geq t_{0} .
\end{aligned}
$$

where $\mu_{1}$ is the weight of each of these cone-hysterons such that

$$
\sum_{i=1}^{n} \mu_{i}=1,
$$

and where $\left(\alpha_{\mathrm{j}}, \beta_{\mathrm{j}}\right)$ are the threshold values for a particular cone-hysteron $C_{\alpha_{j}, \beta_{j}}$.

Here too an algorithmic definition for Eqn.(11) can be written as

$$
\begin{aligned}
& y(t)=\sum_{i=1}^{n} \mu_{i} \frac{s(t)}{k_{\text {new }_{i}}}, \\
& k_{\text {new }_{i}}=\left\{\begin{array}{cc}
k_{2}, & s(t)>\beta_{i} \\
k_{1}, & s(t)<\alpha_{i} \\
k_{\text {old }_{i}}, & \alpha_{i} \leq s(t) \leq \beta_{i}
\end{array},\right.
\end{aligned}
$$

The simple case where all of these cone-hysterons have equal weighting will be used

$$
\mu_{i}=\frac{1}{n}, \quad i=1, \ldots, n \text {. }
$$

where $n$ is the number of cone-hysterons $C_{\alpha_{i}, \beta_{i}}$. This can be illustrated by taking an arbitrary number of equally weighted cone-hysterons and using Eqn. 14 to construct the output shown in Fig. 18. The cone-hysterons used to produce Fig. 18 are represented on a Preisach-plane shown in Fig. 19. Note the arbitrary values of $\left(\alpha_{i}, \beta_{i}\right)$.

Some of the features that should be expected from the continuous case can now be seen; when the number of

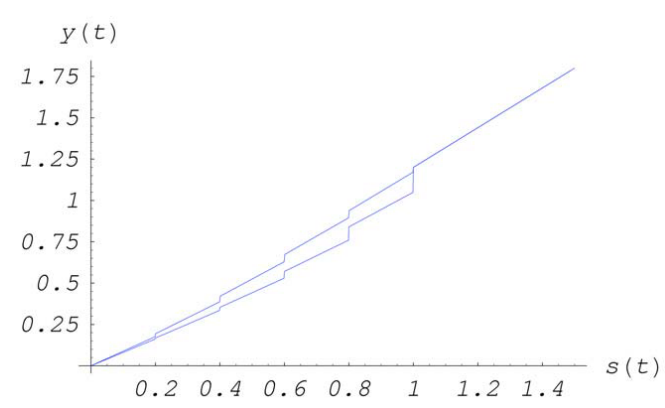

Fig. 18. Discrete hysteresis loop generated by 21 cone-hysterons.

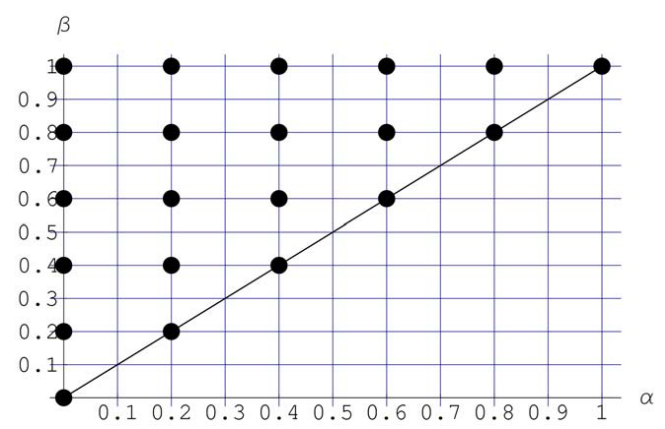

Fig. 19. Preisach plane with 21 discrete cone-hysterons

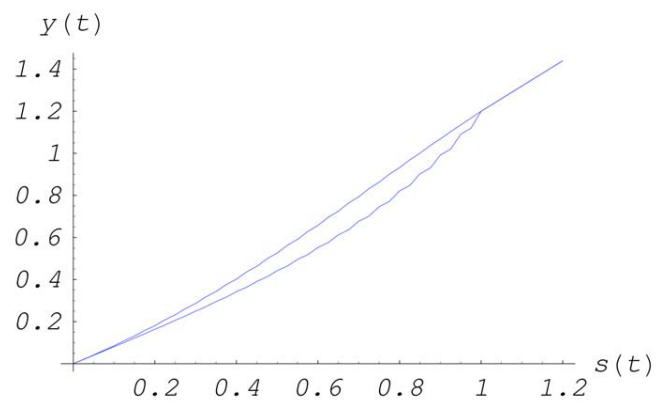

Fig. 20. Discrete hysteresis loop generated by 231 cone-hysterons.

hysterons is increased a smoother result should be expected; this can see the by comparing Fig. 18 with Fig. 20.

In the continuous case the number of cone-hysterons increases to infinity, $n \rightarrow \infty$. As with the discrete case, the output from the continuous case is confined between the two lines $y=\frac{s}{k_{1}}$ and $y=\frac{s}{k_{2}}$. When the cone-hysterons are all switched to $k_{2}$ i.e. when Eqn.(14) becomes:

$$
y(t)=\frac{1}{n} \sum_{i=1}^{n} \frac{s(t)}{k_{2}} .
$$

These cone-hysterons can only be switched to $k_{2}$ when $s(t)>\beta_{\max }$ where $\beta_{\max }$ is the largest possible threshold for all hysterons. Hence Eqn.(15) becomes 


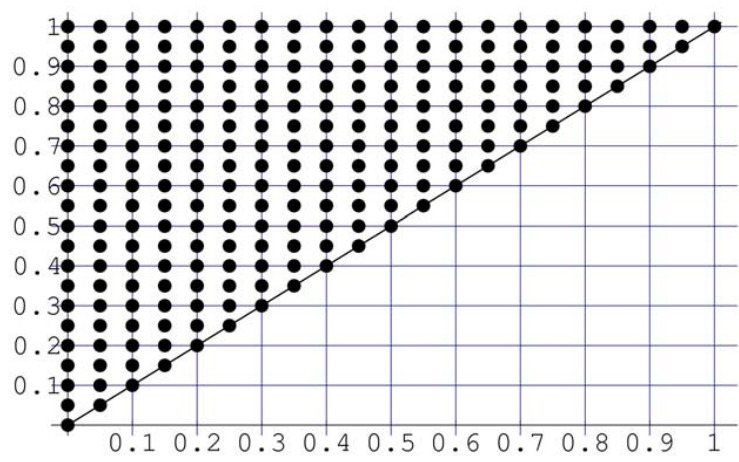

Fig. 21. Preisach plane with 231 discrete cone-hysterons.

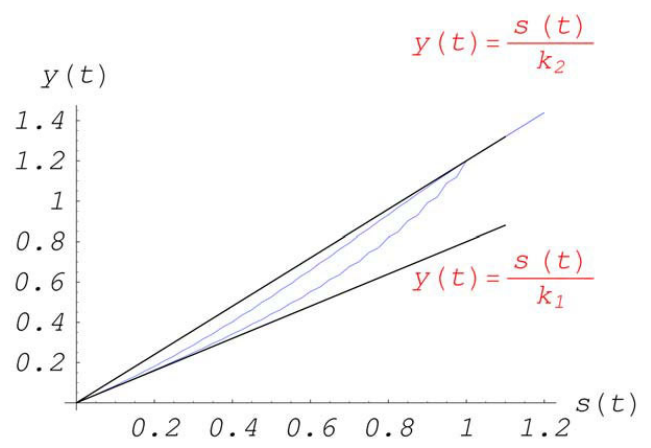

Fig. 22. Parallel connection of 231 cone-hysterons with lines of maximum and minimum possible slopes.

$$
y(t)=\frac{s(t)}{k_{2}}, \quad s(t) \geq \beta_{\max },
$$

which is the equation of the upper line of the cone as shown in Fig. 22. Likewise when all hysterons are switched to $k_{1}$ i.e.

$$
y(t)=\frac{1}{n} \sum_{i=1}^{n} \frac{s(t)}{k_{1}},
$$

which can only occur when $s(t) \leq \alpha_{\min }$, where $\alpha_{\min }$ is the smallest possible threshold for all hysterons. Hence Eqn. (17) becomes

$$
y=\frac{s(t)}{k_{1}}, \quad s(t) \leq \alpha_{\text {min }},
$$

which is the equation of the lower line of the cone as shown in Fig. 22. Further details are given in Appendix A.

\section{RESULTS}

The Operator ordinary differential equation (ODE) for the hysteretic linear reservoir

$$
\frac{d s}{d t}=x(t)-P\{s(t)\}
$$

(see Appendix A for its derivation) was numerically solved for $s(t)$ using a numerical algorithm. The algorithm comprised of a standard four-step Runge-Kutta (RK4) integrator, and a Preisach operator algorithm, which was developed by Rasskazov in Brokate et al. (2005) and has been used in work by Flynn and Rasskazov (2005), and Donegan (2004).

The input $x(t)$ to the hysteretic linear reservoir for the numerical experiments was a damped sinusoid of the following type:

$$
x(t)=A e^{-\kappa t}\left(1-\cos \left(\frac{2 \pi t}{T}\right)\right),
$$

where $A$ was the amplitude, $\kappa$ was the damping constant and $T$ was the period. A uniform density in the Preisach half-plane has been used in each.

Figures 23 and 25 show plots of the inflow $y(t)$ against the mass in storage $s(t)$ for the anticlockwise and clockwise cases respectively. The relationship in both cases is strongly hysteretic.

Figures 24 and 26 show the time series plots of the input $x(t)$, output $y(t)$, solution $s(t)$ to the ODE and the right hand side of the ODE: $x(t)-y(t)$ for the given sinusoidal input Eqn. 20.

Whether the hysteretic linear reservoir is of use in hydrology must await the examination of hydrological timeseries for the presence of hysteresis at larger spatial scales.

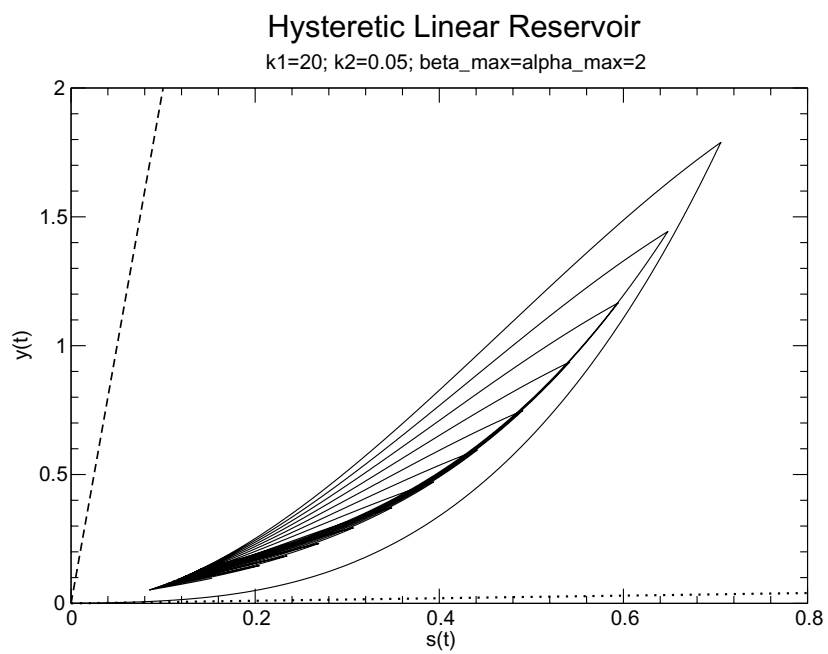

Fig. 23. Anti-clockwise hysteresis loops generated from a plot of $y(t)$ against $s(t)$ and with an exponentially decreasing input. 

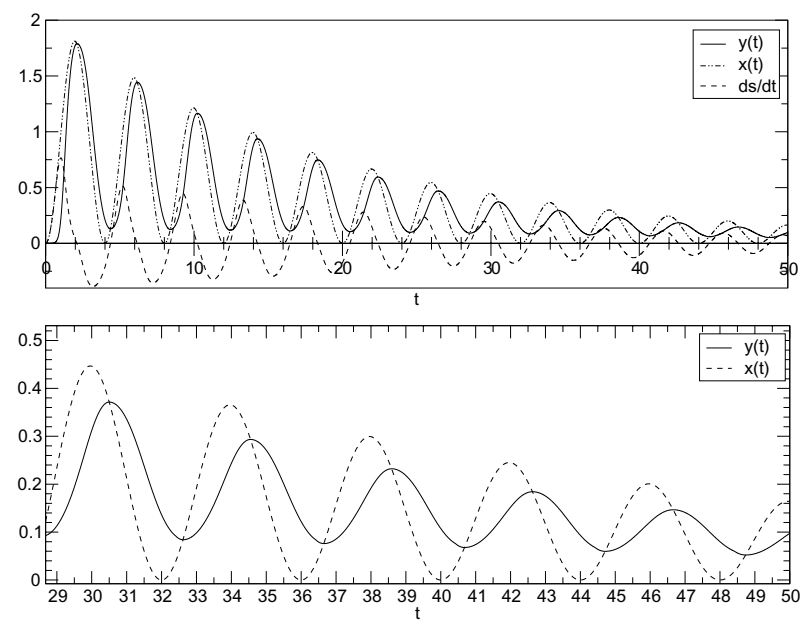

Fig. 24. Time series for exponentially decreasing input $x(t)$. ConeHysteron parameters $k_{1}=20$ and $k_{2}=0.05$.

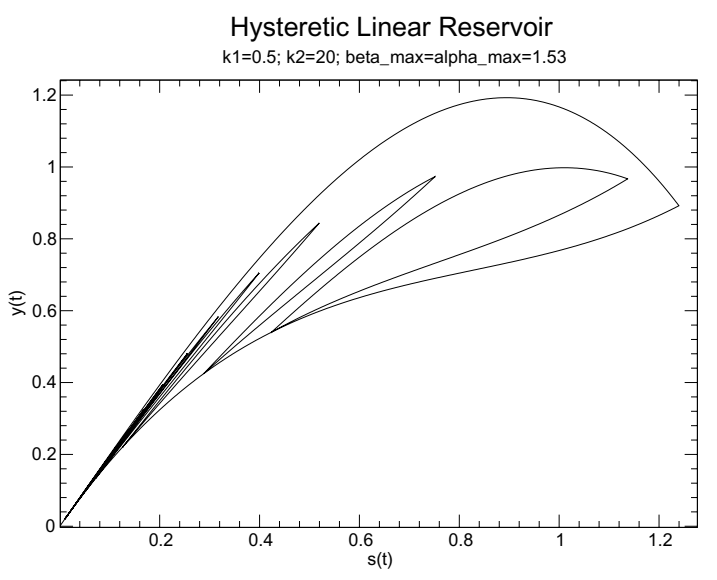

Fig. 25. Clockwise hysteresis loops generated from a plot of $y(t)$ against $s(t)$.
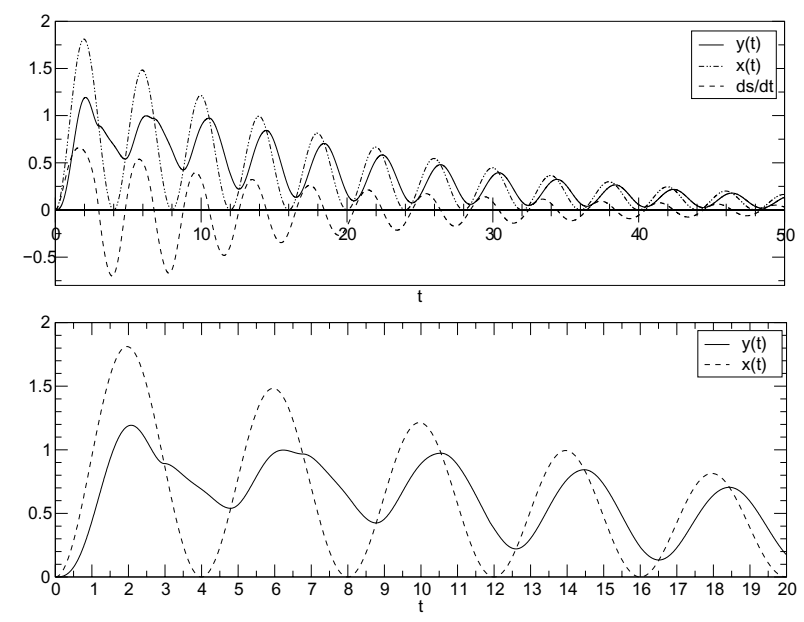

Fig. 26. The upper graph shows time-series plots of $y(t), x(t)$ and $\frac{d s(t)}{d t}$ against $t$. The lower graph shows a magnified region of a time-series plot of $y(t)$ against $t$. The Cone-Hysteron parameters are $k_{1}=0.5, k_{2}=20$ and $\beta_{\max }=\alpha_{\max }=1.53$.

\section{Conclusions}

Hysteresis is the collective name for rate-independent nonlinearities that are expressed through thresholds, switches and branches. These cannot be removed by means of smooth transformations of the system variables. Exceedance of a threshold, or the occurrence of a turning point in the input, switches the output onto a particular output branch. Rateindependent branching on a very large set of switches with non-local memory is the central concepts in the new definition of hysteresis. Hysteretic loops are a special case. A non-linear systems theory has been developed for the selfconsistent mathematical description of hydrological systems that exhibit hysteresis. Two results have been presented: a conceptual model for the hysteretic soil-moisture characteristic at the pedon scale and a hysteretic linear reservoir at the catchment scale. Both are based on the Preisach model, which is a weighted parallel connection of rate-independent hysterons, or atoms of hysteresis. While each hysteron has local memory, many hysterons in parallel can have non-local memory.

A result of particular significance is the demonstration that the independent domain model of the soil moisture characteristic due to Childs, Poulavassilis, Mualem and others, is equivalent to the Preisach model, a result reminiscent of the reduction of the theory of the unit hydrograph to linear systems theory in the 1950s. A significant reduction in the number of model parameters has also been achieved.

This work also extends the modelling paradigm of Forrester (1968) to highly non-linear systems that exhibit hysteresis. The conservation laws of mass, momentum and energy, when expressed in integral form, always exhibit local memory and rate dependence. Consequently, hysteresis cannot be introduced into them directly. The place to insert non-local memory, hysterons, switches, thresholds and branches is in the rate-independent relationships that close the conservation laws. The introduction of the Preisach model into the classic linear reservoir to make it exhibit non-local memory and hysteresis illustrates the extended paradigm.

\section{Acknowledgements}

The authors acknowledge the many fruitful discussions with Alexei Pokrovskii, Dmitrii Rachinskii and James Gleeson. This work has been funded by SFI grant 05/RFP/ENG0062 and the BCRI (Boole Centre for Research in Informatics). 


\section{References}

Brokate, M. and Sprekels, J., 1996. Hysteresis and phase transitions, vol. 121, Applied Mathematical Sciences, SpringerVerlag, New York, USA.

Brokate, M., Pokrovskii, A., Rachinskii, D. and Rasskazov, O., 2005. Differential equations with hysteresis via a canonical example. In: Science of Hysteresis, vol. 1, Academic Press, Oxford, UK.127-291.

Cross, R., 1995. Hysteresis, The Handbook of Economic Methodology, Edward Edgar.

Cross, R. and Allan, A., 1988. On the hystory of hysteresis. In: Unemployment, Hysteresis \& Natural Rate Hypothesis, R. Cross, (Ed.), Blackwell, Oxford, UK. 26-38.

de Gennes, P.-G., Brochard-Wyart, F. and Quere, D., 2003. Capillarity and Wetting Phenomena: Drops, Bubbles, Pearls, Waves. Springer, New York, USA.

Donegan, M., 2004. Semi-linear duffing equation with discrete Preisach non-linearity. MSc thesis, University College, Cork, Ireland.

Dooge, J. and O'Kane, P., 2003. Deterministic Methods in Systems Hydrology. A.A. Balkema Publishers, Lisse, The Netherlands.

Ewing, J. A., 1885. Experimental research in magnetism. Trans. Roy. Soc., London, 176, II, 523-640.

Flynn,D., 2003. Soil moisture hysteresis, http://euclid.ucc.ie/ appliedmath/soilhyst/node $17 . h$ tm

Flynn, D., 2004. Appication of the Preisach model in soil-moisture hysteresis. MSc thesis, University College Cork, Cork, Ireland

Flynn, D. and O'Kane, P., 2004. A review of hysteresis in soil physics. Geophys. Res. Abs., EGU04-A-07314, 6, 514-520.

Flynn, D. and Rasskazov, O., 2005. On the integration of an ODE involving the derivative of a Preisach nonlinearity. J. Phys. Conf. Ser., 22, 43-55.

Flynn, D., McNamara, H., O'Kane, P. and Pokrovskii, A., 2005. Application of the Preisach model to soil-moisture hysteresis. In: Science of hysteresis, vol. 3, Academic Press, Oxford, UK. 689-744

Forrester, J., 1968. Principles of Systems. Wright-Allen Press, Cambridge,MA, USA.

Haines, W. B., 1930. Studies in the physical properties of soil. V. The hysteresis effect in capillary properties, and the modes of moisture distribution associated therewith. J. Agr. Sci., 20, 97116.

Haverkamp, R., Reggiani, P., Ross, P. and Parlange, J., 2002. Soil Water hysteresis prediction model based on theory and geometric scaling. In: Enviromental Mechanics : Water, Mass and Energy Transfer in the Biosphere. Geophysical Monograph 129., Amer. Geophys. Un., 213-246.

Holland, F., 2005. Dept. of Mathematics, UCC, Ireland. Personal communication.

Krasnosel'skii, M. and Pokrovskii, A., 1989. Systems with Hysteresis. Springer-Verlag, New York, (translated from the Russian by Marek Niezgódka).

Krejci, P., 1996. Hysteresis, Convexity and Dissipation in Hyperbolic Equations, Gakkotosho, Tokio.

Krejci, P. and Sprekels, J., 1999. Strong solutions to equations of visco-thermo-plasticity with a temperature-dependent hysteretic strain-stress law. In: Variations of domain and free-boundary problems in solid mechanics (Paris, 1997). Solid Mech. Appl., 66,. 237-244, Kluwer Acad. Publ., Dordrecht.

Krejci, P., O'Kane, J. and Pokrovskii, A., 2004. Periodic solutions to a soil model with hysteretic hydrology. Geophys. Res. Abs., vol. 6, 01872, EGU 1st General Assembly Nice, France.
Mayergoyz, I., 1991. Mathematical Models of Hysteresis. Springer-Verlag, New York, USA.

Mayergoyz, I., 2003. Mathematical models of hysteresis and their applications, chap. 1 [of what?], Elsevier, Amsterdam, The Netherlands

Mualem, Y., 1974a. A conceptual model of hysteresis. Water Resour. Res., 10, 514-520.

Mualem, Y., 1984. A modified dependent-domain theory of hysteresis. Soil Sci., 137, 283-291.

Mualem, Y. and Dagan, G., 1975. Dependent domain model of capillary hysteresis. Water Resour. Res., 11, 452-460.

Mualem, Y. and Miller, E.E., 1979. Hysteresis model based on an explicit domain-dependence function. Soil Sci. Soc. Amer. J., 43, 1067-1073

Mualem, Y. and Morelseytoux, H.J., 1979. Analysis of a capillary hysteresis model based on a one-variable distribution function. Water Resour. Res., 14, 605-610.

O'Kane, J., 1991. Implications for remote sensing of natural switching from atmosphere-controlled to soil-controlled evaporation or enfiltration. In: Land Surface Evaporation: Measurement and Parameterization, Springer Verlag, New York, USA. 371-381.

O'Kane, J., 2004. Hysteresis, the missing non-linearity. Discussion of "Bringing it all together" by J.C.I. Dooge. Hydrol. Earth Syst. Sci. Discuss., 1, 41-73.

O'Kane, J.P., 2006. The hysteretic linear reservoir-a new Preisach model. Physica B: Condensed Matter, 372, 388-392.

O'Kane, J., 2005. Hysteresis in hydrology. Acta Geophys, Pol., 53, 373-383.

O'Kane, J., Pokrovski, A., Krejci, P. and Haverkamp, R., 2003 a. Hysteresis and terrestrial hydrology. EGS-AGU-EUG Joint Assembly, Nice, France. Abstract no: P0605; EAE03-A-06154; HS1-1MO2P-0605

O'Kane, J., Pokrovski, A., Krejci, P. and Haverkamp, R., 2003 b. Application of the Preisach model to soil-moisture hysteresis. Eos Trans. AGU, 84(46), Fall Meet. Suppl., Abstract H22J-06.

Parlange, J.-Y., 1980. Water transport in soils. Ann. Rev. Fluid Mech., 12, 77 - 102.

Poulovassilis, A., 1962. Hysteresis of pore water, an application of the concept of independent domains. Soil Sci., 93, 405-412.

Poulovassilis, A., 1969. Effect of hysteresis of pore-water on hydraulic conductivity. J. Soil Sci., 20, 52-56.

Poulovassilis, A., 1970a. Effect of entrapped air on hysteresis curves of a porous body and on its hydraulic conductivity. Soil Sci., 109, 154-162.

Poulovassilis, A., 1970b. Hysteresis of pore water in granular porous bodies. Soil Sci., 109, 5-12.

Poulovassilis, A. and Childs, E. C., 1971. Hysteresis of pore water - non-independence of domains. Soil Sci., 112, 301-312.

Poulovassilis, A. and Tzimas, E., 1974. Hysteresis in relationship between hydraulic conductivity and suction. Soil Sci., 117, 250256.

Preisach, P., 1935. Über die magnetische Nachwirkung. Zeitschrift für Physik, 94, 277-302.

Rasskazov, O., 2002. Systems with hysteresis. http://euclid.ucc.ie/ hysteresis/node17.htm

Visintin, A., 1994. Differential Models of Hysteresis. Springer, Berlin, Germany. 


\section{Appendix A}

\section{Derivation of the operator differential equation with Preisach hysteresis}

The history of the system is recorded as a 'staircase' on a Preisach half plane. This 'staircase' divides the hysterons into two independent regions $S^{+}$and $S^{\llcorner}$, where the hysterons are switched on or off respectively see Fig. 27. The output in that case is

$$
y(t)=1 \times m\left(S^{+}\right)+0 \times m\left(S^{-}\right),
$$

where $m$ is the measure of a particular region. The measure is simply the area of a particular region of the plane but it can be extended to cover the case where the measure is nonuniform, which was the case for the Wedge Model (Flynn et al., 2005).

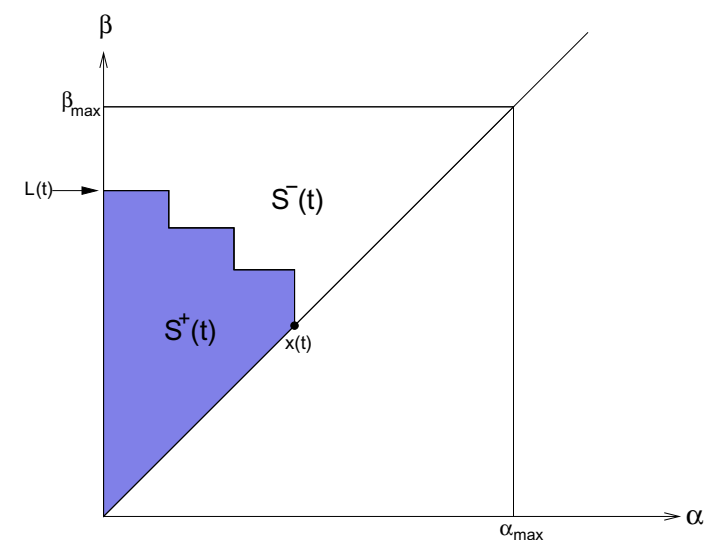

Fig. 27. Shown above is a representation of the Classical Preisach Half-Plane. The boundaries are $(0,0)$ and $\alpha_{\max }, \beta_{\max }$. The staircase $L(t)$ divides the half plane into two regions $S^{+}(t)$ and $S(t)$. The region $S^{+}(t)$ is where the hysterons are all switched on i.e. 1 and the region $S^{-}(t)$ is where the hysterons are all switched off i.e. 0 . Note that $L, S^{+}$and $S$ are all time dependent, as the staircase division $L(t)$ between the two regions evolves over time as the point $x(t)$ moves, hence the regions $S^{+}$and $S$ either grow or shrink.

For a Preisach model with cone-hysterons, the output must have a different form as the states $\frac{1}{k_{1}}$ and $\frac{1}{k_{2}}$ must be recorded on the half-plane. This can be achieved by modifying the Preisach-plane as shown in Fig. 28. From this it can be seen that the output will the following

$$
y(t)=\left(\frac{m\left(S_{k 1}\right)}{k_{1}}+\frac{m\left(S_{k 2}\right)}{k_{2}}\right) s(t),
$$

where $S_{k 1}$ and ${ }_{k 2}$ are the regions (see Fig. 28) where hysterons are switched to $k_{1}$ or $k_{2}$ respectively, and $s(t)$ is the current input variable. This can also be represented in a block diagram shown in Fig. 29.

Since $0 \leq s(t) \leq \beta_{\max }$ then Eqn. (22) can be simplified

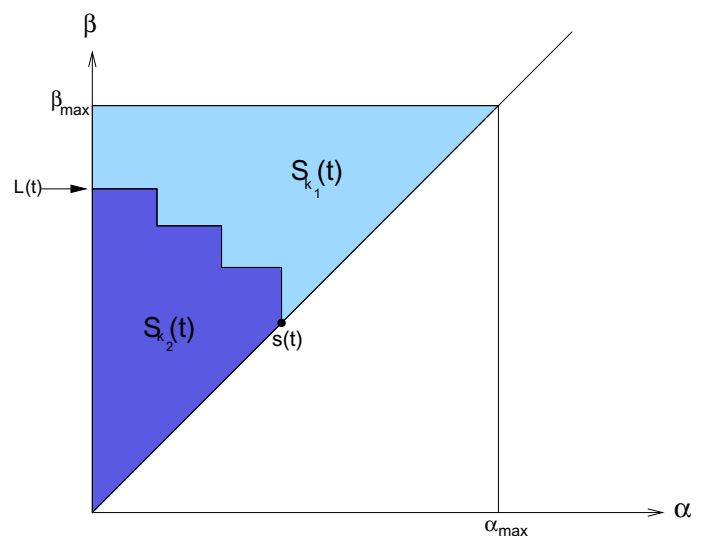

Fig. 28. Shown above is a modified Preisach-Plane. The boundaries are $(0,0)$ and $\left(\alpha_{\max }, \beta_{\max }\right)$. The staircase $L(t)$ divides the half plane into two regions $S_{k 1}(t)$ and $S_{k 2}(t)$. The region $S_{k 1}(t)$ contains the hysterons which have their $k$-values switched to $k_{1}$ and the region $S_{k 2}(t)$ contains hysterons with their $k$-values switched to the $k_{2}$. Note that $L, S^{+}$and $S$ are all time dependent, as the staircase division $L(t)$ between the two regions evolves over time as the point $x(t)$ moves, hence the regions $S^{+}$and $S$ either grow or shrink.

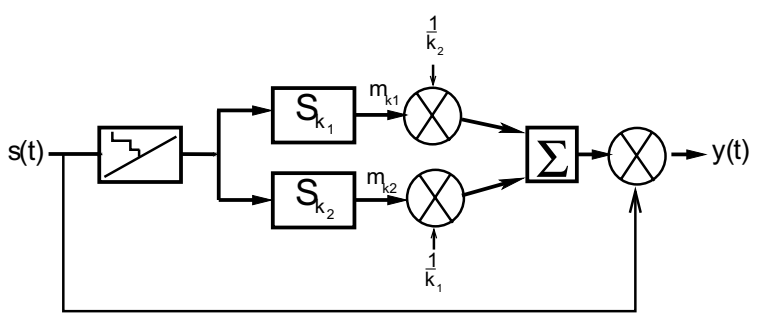

Fig. 29. A schematic representation of Eqn. (35).

further by using the following

$$
\begin{aligned}
& m(S) \quad m\left(S_{k 1}\right)+m\left(S_{k 2}\right), \\
& \Rightarrow m\left(S_{k 2}\right)=m(S)-m\left(S_{k 1}\right) .
\end{aligned}
$$

Eqn. (22) can be rewritten as

$$
\begin{aligned}
& y(t)=\left[\frac{m\left(S_{k 1}\right)}{k_{1}}+\frac{m(S)-m\left(S_{k 1}\right)}{k_{2}}\right] s(t), \\
& =\left[\Omega m\left(S_{k 1}\right)+\frac{m(S)}{k_{2}}\right] s(t),
\end{aligned}
$$

where $\Omega=\left(\frac{1}{k_{1}}+\frac{1}{k_{2}}\right)$. This can be written in operator form as

$$
y(t)=P\{s(t)\}
$$

where $P$ is the Preisach operator. The linear reservoir Eqn.(5) with hysteresis is now an operator differential equation

$$
\frac{d s}{d t}=x(t)-P\{s(t)\}
$$

